\title{
The Julia-Wolff-Carathéodory theorem(s)
}

\author{
by Marco Abate $^{1}$ and Roberto Tauraso ${ }^{2}$ \\ ${ }^{1}$ Dipartimento di Matematica, Università di Roma "Tor Vergata", Via della Ricerca Scientifica, 00133 Roma, Italy \\ 2 Dipartimento di Matematica "Ulisse Dini", Università di Firenze, Viale Morgagni 67/A, 50134 Firenze, Italy
}

November 1997

A classical result in the theory of one complex variable is Fatou's theorem:

Theorem 1: (Fatou $[\mathrm{F}])$ Let $f \in \operatorname{Hol}(\Delta, \Delta)$ be a holomorphic self-map of the unit disk $\Delta$ in the complex plane. Then $f(\zeta)$ has non-tangential limit at a.e. point $\sigma \in \partial \Delta$.

As satisfying as it is from several points of view, this theorem leaves open the question of what happens at a specific point $\sigma_{0} \in \partial \Delta$. Of course, to get a sensible statement one needs to make some assumptions on the function $f$. In 1920, Julia ([Ju]) identified the right hypotheses, showing how to get the existence of the non-tangential limit at a given boundary point using Schwarz's lemma. But the real breakthrough is due to Wolff ([W]) in 1926 and Carathéodory ([C]) in 1929, who proved that under Julia's hypotheses the derivative too admits non-tangential limit at the specified boundary point. Their results are collected in the following statement, the Julia-Wolff-Carathéodory theorem:

Theorem 2: (Julia-Wolff-Carathéodory) Let $f \in \operatorname{Hol}(\Delta, \Delta)$ and $\sigma_{0} \in \partial \Delta$ be such that

$$
\liminf _{\zeta \rightarrow \sigma_{0}} \frac{1-|f(\zeta)|}{1-|\zeta|}=\alpha<+\infty
$$

Then:

(i) $f$ has non-tangential limit $\tau_{0} \in \partial \Delta$ at $\sigma_{0}$;

(ii) $f^{\prime}$ has non-tangential limit $\alpha \tau_{0} \overline{\sigma_{0}}$ at $\sigma_{0}$.

See, e.g., [A2] for proofs and applications. By the way, it should be remarked that the liminf in (1) is always strictly positive.

The aim of this note is to describe a general framework allowing the generalization of the Julia-Wolff-Carathéodory theorem (in short, the JWC theorem) to several classes of bounded domains in $\mathbb{C}^{n}$. The framework is known to work at least in the following cases: the unit ball $B^{n}$ of $\mathbb{C}^{n}$ (Hervé $[\mathrm{H}]$ and Rudin $[\mathrm{R}]$ ); strongly convex domains ([A1]); strongly pseudoconvex domains ([A3]); polydisks (Jafari [J], and [A4]); and some complex ellipsoids ([AT]). All the needed ingredients are available in general weakly convex domains, and so it might be valid in that setting too.

The main idea, in the spirit of Krantz's generalizations of Fatou's theorem (see [Kr]), is that the boundary behavior of holomorphic functions defined on (or with values in) a bounded domain must be controlled by the boundary behavior of the intrinsic Kobayashi distance and metric of the domain. To substantiate this idea, we need a way to define a suitable generalization to several variables of the notion of non-tangential limit, generalization expressed in terms of the Kobayashi metric and distance. 
Actually, it turns out that two such generalizations will be needed: one defined via approach regions (similar to what Korányi, Stein, Krantz and others have done, but not exactly the same); and a second one defined via approaching curves (similar to what Čirka, Cima-Krantz and others have done, but not exactly the same).

But first of all let us recall the definition of the Kobayashi metric and distance (see, e.g., [A2, JP] for details, proofs, history and much more). Let $X$ be a complex manifold; the Kobayashi (pseudo)metric $\kappa_{X}: T X \rightarrow \mathbb{R}^{+}$is defined by

$$
\kappa_{X}(z ; v)=\inf \left\{|\xi| \mid \exists \varphi \in \operatorname{Hol}(\Delta, X): \varphi(0)=z, d \varphi_{0}(\xi)=v\right\}
$$

for all $z \in X$ and $v \in T_{z} X$. This is a (in general only upper semicontinuous, often continuous, almost never hermitian) Finsler metric on $X$, that is it satisfies

$$
\kappa_{X}(z ; \lambda v)=|\lambda| \kappa_{X}(z ; v)
$$

for all $z \in X, v \in T_{z} X$ and $\lambda \in \mathbb{C}$. Its main property is that it is contracted by holomorphic maps: if $f \in \operatorname{Hol}(X, Y)$ and $(z ; v) \in T X$ then

$$
\kappa_{Y}\left(f(z) ; d f_{z}(v)\right) \leq \kappa_{X}(z ; v) .
$$

In particular, Schwarz's lemma implies that $\kappa_{\Delta}$ is the Poincaré metric on $\Delta$-and thus the Kobayashi metric can be regarded as a natural generalization of the Poincaré metric to $n$-dimensional complex manifolds.

The Finsler property (2) allows one to use $\kappa_{X}$ to measure the length of curves, exactly as in Riemannian geometry. The infimum of the (Kobayashi) length of curves connecting two points gives the Kobayashi (pseudo)distance $k_{X}: X \times X \rightarrow \mathbb{R}^{+}$of $X$. It might happen that $k_{X}\left(z_{1}, z_{2}\right)=0$ even though $z_{1} \neq z_{2}$ (for instance, it is easy to check that $k_{\mathbb{C}^{n}} \equiv 0$ ); but if this is not the case, that is if $k_{X}$ is a true distance, we say that $X$ is (Kobayashi) hyperbolic. If moreover $k_{X}$ is a complete distance, then $X$ is complete hyperbolic. For instance, all bounded domains in $\mathbb{C}^{n}$ are hyperbolic, and all convex and strongly pseudoconvex domains are complete hyperbolic.

Again, the Kobayashi distance is contracted by holomorphic maps: if $f \in \operatorname{Hol}(X, Y)$ and $z_{1}, z_{2} \in X$ then

$$
k_{Y}\left(f\left(z_{1}\right), f\left(z_{2}\right)\right) \leq k_{X}\left(z_{1}, z_{2}\right)
$$

In particular, $k_{\Delta}$ is the Poincaré distance of $\Delta$.

The contracting property (which is a built-in Schwarz-Pick lemma) is what makes the Kobayashi metric and distance so useful. Unfortunately, as it often happens with intrinsic objects defined by computing the extremal of some functional, the Kobayashi metric and distance are easy to estimate but very hard to compute explicitly. Anyway, a wealth of informations about them is available, particularly in strongly pseudoconvex and in convex domains; again, see [A2, JP] for details.

Now we can get back to the generalizations of the non-tangential limit. One way of defining the non-tangential limit in the unit disk is using Stolz regions. A Stolz region $S(\sigma, M) \subset \Delta$ of vertex $\sigma \in \partial \Delta$ and amplitude $M>1$ is the egg-shaped region given by

$$
S(\sigma, M)=\left\{\zeta \in \Delta \mid \frac{|\sigma-\zeta|}{1-|\zeta|}<M\right\}
$$


notice that $S(\sigma, M)$ is defined by taking the ratio of the distance $|\sigma-\zeta|$ from $\zeta$ to $\sigma$ with the distance $1-|\zeta|$ from $\zeta$ to the whole boundary of $\Delta$. Then $f: \Delta \rightarrow \mathbb{C}$ has non-tangential limit $L \in \mathbb{C}$ at $\sigma$ iff $f(\zeta) \rightarrow L$ as $\zeta \rightarrow \sigma$ inside any Stolz region of vertex $\sigma$.

Korányi $([\mathrm{K}])$, in 1969, discovered that the right generalization of Stolz regions in the unit ball $B^{n}$ of $\mathbb{C}^{n}$ is not a cone-shaped region but the following set:

$$
K(x, M)=\left\{z \in B^{n} \mid \frac{|1-(z, x)|}{1-\|z\|}<M\right\}
$$

where $x \in \partial B^{n}, M>1,(\cdot, \cdot)$ is the canonical hermitian product of $\mathbb{C}^{n}$, and $\|\cdot\|$ is the euclidean norm. Contrarily to the one-dimensional case, $K(x, M)$ is non-tangential at $x$ only in the direction orthogonal to the boundary, being instead parabolically tangent to $\partial B^{n}$ along the complex-tangential directions.

Using these regions, and their analogues in more general domains, Korányi and Stein ([KS, S]) have successfully generalized Fatou's theorem to several complex variables (see also $[\mathrm{Kr}]$ ); but their definitions were mostly in euclidean terms, and so unsuitable to our needs.

An alternative approach is the following. Let $D \subset \subset \mathbb{C}^{n}$ be a complete hyperbolic domain; fix a base point $z_{0} \in D$, and let $k_{D}$ denote the Kobayashi distance of $D$. If $z \in D$, then $k_{D}(z, w)$ tends to $+\infty$ as $w$ tends to $x \in \partial D$; therefore the normalized number

$$
\limsup _{w \rightarrow x}\left[k_{D}(z, w)-k_{D}\left(z_{0}, w\right)\right]
$$

can be thought of as a (always finite, possibly negative) "distance" from $z \in D$ to $x \in \partial D$ (computing it in the unit disk, for instance, we see that it actually is half the logarithm of such a "distance", explaining the negative values).

Furthermore, in several complete hyperbolic domains (strongly pseudoconvex domains, polydisks, and the like; see, e.g., [A2]) it is known that $k_{D}\left(z_{0}, z\right)$ goes to infinity exactly as $-\frac{1}{2} \log d(z, \partial D)$ as $z$ tends to $\partial D$. Therefore a possible generalization of a Stolz region is given by the $K$-region of vertex $x \in \partial D$, amplitude $M>1$ and pole $z_{0} \in D$ defined by setting

$$
K_{z_{0}}(x, M)=\left\{z \in D \mid \limsup _{w \rightarrow x}\left[k_{D}(z, w)-k_{D}\left(z_{0}, w\right)\right]+k_{D}\left(z_{0}, z\right)<\log M\right\}
$$

notice that changing the pole amounts to a shifting of the amplitudes, and so it is not relevant.

An easy computation shows that in the unit ball $K_{0}(x, M)$ is exactly the set $K(x, M)$ defined by Korányi. Furthermore, it is not difficult to prove (see $[$ A3, 4]) that in strongly pseudoconvex domains our $K$-regions are comparable with Korányi-Stein admissible regions, and that in convex domains they are comparable with Krantz's admissible regions. Therefore, if we say that a function $f \in \operatorname{Hol}\left(D, \mathbb{C}^{m}\right)$ has $K$-limit $L$ at $x \in \partial D$ if $f(z) \rightarrow L$ as $z \rightarrow x$ inside any $K$-region of vertex $x$, we have recovered in terms of the Kobayashi distance only the admissible limit defined by Korányi-Stein in euclidean terms. 
Now, in condition (1) of Theorem $2,1-|\zeta|$ is the euclidean distance $d(\zeta, \partial \Delta)$ of $\zeta$ from the boundary $\partial \Delta$. Recalling again that $k_{D}\left(z_{0}, \cdot\right)$ is comparable to $-\frac{1}{2} \log d(\cdot, \partial D)$, it is then natural to replace (1) by the following condition:

$$
\liminf _{z \rightarrow x}\left[k_{D}\left(z_{0}, z\right)-k_{\Delta}(0, f(z))\right]<+\infty
$$

Indeed, this is exactly what we need to generalize Julia's part of the Julia-Wolff-Carathéodory theorem:

Theorem 3: ([A1]) Let $D \subset \subset \mathbb{C}^{n}$ be complete hyperbolic. Take $f \in \operatorname{Hol}(D, \Delta)$ and $x \in \partial D$ such that

$$
\liminf _{z \rightarrow x}\left[k_{D}\left(z_{0}, z\right)-k_{\Delta}(0, f(z))\right]<+\infty .
$$

Then $f$ has $K$-limit $\tau \in \partial \Delta$ at $x$.

It is worth pointing out that similar statements hold for holomorphic maps $f: D_{1} \rightarrow D_{2}$ under suitable hypotheses on $D_{2}$ (e.g., if $D_{2}$ is strongly pseudoconvex). The proof is just an application of the contracting property of the Kobayashi distance.

Unfortunately, as already remarked by Rudin in the unit ball, the $K$-limit is not suitable for the generalization of the Wolff-Carathéodory part of the JWC theorem. To single out the correct replacement it is necessary to examine another classical one-variable result, the Lindelöf principle:

Theorem 4: $([\mathrm{Li}])$ Let $f \in \operatorname{Hol}(\Delta, \Delta)$ be so that there is a continuous curve $\gamma:[0,1) \rightarrow \Delta$ ending at $\sigma_{0} \in \partial \Delta$ such that $f(\gamma(t)) \rightarrow L \in \mathbb{C}$. Then $f$ has non-tangential limit $L$ at $\sigma_{0}$.

In other words, for a bounded holomorphic function defined on the disk the existence of the limit along a given curve forces the existence of the limit along any non-tangential curve.

It turns out that there are several ways to generalize the Lindelöf principle to several variables. Let $D \subset \subset \mathbb{C}^{n}$; if $x \in \partial D$, an $x$-curve is a continuous curve $\gamma:[0,1) \rightarrow D$ such that $\gamma(t) \rightarrow x$ as $t \rightarrow 1^{-}$. Then a Lindelöf principle is a statement of the following kind:

"Let $x \in \partial D$. Then there are a class $\mathcal{S}$ of $x$-curves and a subclass $\mathcal{R} \subseteq \mathcal{S}$ such that the following holds: for every $f \in \operatorname{Hol}(D, \Delta)$, if there is a curve $\gamma^{o} \in \mathcal{S}$ such that $f\left(\gamma^{o}(t)\right) \rightarrow L \in \mathbb{C}$ as $t \rightarrow 1^{-}$, then $f(\gamma(t)) \rightarrow L$ as $t \rightarrow 1^{-}$for all $\gamma \in \mathcal{R}$."

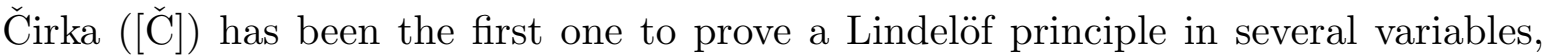
defining classes $\mathcal{S}$ and $\mathcal{R}$ in euclidean terms in domains with $C^{2}$ boundary. Later on, Cima and Krantz $([\mathrm{CK}])$ obtained another Lindelöf principle using both the euclidean structure and the Kobayashi distance to define the classes $\mathcal{S}$ and $\mathcal{R}$. In [A1] another approach has been suggested, using projection devices.

Let $D \subset \mathbb{C}^{n}$ be a domain. A projection device at $x \in \partial D$ is given by: a neighborhood $U$ of $x$ in $\mathbb{C}^{n}$; a holomorphic embedded disk $\varphi_{x}: \bar{\Delta} \rightarrow \bar{D} \cap U$, where $\varphi_{x}$ is holomorphic in the interior and continuous up to the boundary, with $\varphi_{x}(\Delta) \subset D$ and $\varphi_{x}(1)=x$; a device associating to every $x$-curve $\gamma$ in $D \cap U$ an $x$-curve $\gamma_{x}$ in $\varphi_{x}(\Delta)$, that is, a 1-curve $\tilde{\gamma}_{x}=\varphi_{x}^{-1} \circ \gamma_{x}$ in $\Delta$. 
Example 1: Assume that $D$ is strongly convex. Then the euclidean projection device at $x \in \partial D$ is obtained by taking as $U$ the whole of $\mathbb{C}^{n}$, as $\varphi_{x}$ a suitable parametrization of the normal section $D \cap\left(x+\mathbb{C} \mathbf{n}_{x}\right)$ of $D$ at $x$ (where $\mathbf{n}_{x}$ is the unit normal to $\partial D$ at $x$ ), and as $\gamma_{x}$ the orthogonal projection of $\gamma$ into the normal section.

Example 2: Assume that $D$ is convex. Then the canonical projection device at $x \in \partial D$ is obtained by taking as $U$ again the whole of $\mathbb{C}^{n}$, as $\varphi_{x}$ a complex geodesic with $\varphi_{x}(1)=x$, and setting $\gamma_{x}=p_{x} \circ \gamma$, where $p_{x}$ is a holomorphic retraction of $D$ onto the image of $\varphi_{x}$ (a complex geodesic is a holomorphic isometry from $\Delta$ endowed with the Poincaré distance to $D$ endowed with the Kobayashi distance; see [L, A2, JP] for background on complex geodesics and holomorphic retractions).

Example 3: Assume that $D$ is strongly pseudoconvex (or, more generally, locally convexifiable at the boundary). Then a projection device at $x \in \partial D$ is obtained by localizing the previous examples, that is by taking $U$ so that $U \cap D$ is biholomorphic to a convex domain, and then using the $\varphi_{x}$ and $\gamma_{x}$ provided by either the euclidean or the canonical projection device.

Given a projection device, we can define the classes $\mathcal{S}$ and $\mathcal{R}$ mentioned earlier as follows: we shall say that an $x$-curve $\gamma$ is special if $k_{D}\left(\gamma(t), \gamma_{x}(t)\right) \rightarrow 0$ as $t \rightarrow 1^{-}$; and that $\gamma$ is restricted if $\tilde{\gamma}_{x}$ is a non-tangential 1-curve. Then the class $\mathcal{S}$ is the set of all special $x$-curves, while class $\mathcal{R}$ is the set of all special restricted $x$-curves. Finally, we shall say that a holomorphic map $f \in \operatorname{Hol}\left(D, \mathbb{C}^{m}\right)$ has restricted $K$-limit $L$ at $x$ if $f(\gamma(t)) \rightarrow L$ as $t \rightarrow 1^{-}$for all $\gamma \in \mathcal{R}$.

It turns out that we have a Lindelöf principle no matter which projection device we choose:

Theorem 5: ([A1]) Let $D \subset \mathbb{C}^{n}$ be a domain, and choose a projection device at a point $x \in \partial D$. Let $f \in \operatorname{Hol}(D, \Delta)$ be a bounded function such that $f\left(\gamma^{o}(t)\right) \rightarrow L \in \mathbb{C}$ as $t \rightarrow 1^{-}$for a given special $x$-curve $\gamma^{o} \in \mathcal{S}$. Then $f$ has restricted $K$-limit $L$ at $x$.

If $D$ is strongly pseudoconvex and we use a projection device provided by Example 3, it is not difficult to see that a special restricted curve approaches the boundary non-tangentially in the normal direction, and asymptotically less than parabolically in the complex tangential directions. In particular, a function having restricted $K$-limit $L$ at $x \in \partial D$ necessarily has non-tangential limit $L$ at $x$. On the other hand, if we use the projection device provided by Example 2 it turns out that a special restricted curve approaches the boundary asymptotically like the boundary of $K$-regions; in particular, a function having $K$-limit $L$ at $x \in \partial D$ necessarily has restricted $K$-limit $L$ at $x$ (but the converse does not hold: see [R, A4] for the relevant counterexamples).

So we have a huge family of Lindelöf principles. Unfortunately, they are not enough for the generalization of the JWC theorem, because not all the functions needed there are bounded. But there is a replacement: we shall say that a map $f: D \rightarrow \mathbb{C}^{m}$ is $K$-bounded at $x \in \partial D$ if it is bounded in every $K$-region of vertex $x$ (with the bound depending on the amplitude of the $K$-region). Then we have the following:

Theorem 6: Let $f \in \operatorname{Hol}(D, \mathbb{C})$ be $K$-bounded at $x \in \partial D$. Assume that $f\left(\gamma^{o}(t)\right) \rightarrow L$ as $t \rightarrow 1^{-}$for a special restricted $x$-curve $\gamma^{o} \in \mathcal{R}$, where we are using a projection device 
given by Example 2 or Example 3. Then $f$ has restricted $K$-limit $L$ at $x$.

We did not state the exact hypotheses on the domain $D$ because at present we are not yet able to give a general proof of this statement, but only case-by-case arguments. We can prove it for strongly (pseudo)convex domains [A1, 3], polydisks [A4] and some complex ellipsoids $[\mathrm{AT}]$; the structure of the proof is always the same, but the technical details involved seem to depend on the actual geometry of the domain - which is a pity. One of the main open problems in this subject is exactly obtaining a general proof of Theorem 6 .

We finally have all the necessary ingredients to state the generalization of the WolffCarathéodory part of the JWC Theorem. Take $f \in \operatorname{Hol}(D, \Delta)$ so that (4) holds at $x \in \partial D$. We know, by Theorem 3, that $f$ has $K$-limit $\tau \in \partial \Delta$ at $x \in \partial D$; we want to know what happens to the partial derivatives of $f$.

There is an obvious observation to be made: the behavior of the partial derivative

$$
\frac{\partial f}{\partial v}=\sum_{j=1}^{n} v_{j} \frac{\partial f}{\partial z_{j}}
$$

might depend on the vector $v$. For instance, one could expect different behaviors according to $v$ being transversal or complex tangential to the boundary. Actually, it turns out that the behavior of $\partial f / \partial v$ is controlled by the behavior of the Kobayashi metric $\kappa_{D}(z ; v)$. Namely,

Theorem 7: Let $D \subset \subset \mathbb{C}^{n}$ be a "good" domain endowed with a suitable projection device (see below) at $x \in \partial D$. Let $f \in \operatorname{Hol}(D, \Delta)$ be such that

$$
\liminf _{z \rightarrow x}\left[k_{D}\left(z_{0}, z\right)-k_{\Delta}(0, f(z))\right]<+\infty
$$

for one (and hence all) $z_{0} \in D$. Take $v \in \mathbb{C}^{n}$, and let $s \in \mathbb{R}^{+}$be such that

$$
\kappa_{D}(z ; v)=O\left(\frac{1}{d(z, \partial D)^{s}}\right)
$$

as $z \rightarrow x$ in $K$-regions of vertex $x$. Then the function

$$
d(z, \partial D)^{s-1} \frac{\partial f}{\partial v}(z)
$$

has restricted $K$-limit at $x$.

For instance, if $D$ is strongly pseudoconvex, then $s=1 / 2$ if $v$ is complex tangential to $\partial D$ at $x$, and $s=1$ otherwise. On the other hand, if $D=\Delta^{n}$ then $s=1$ always.

A "good" domain, roughly speaking, is a locally convexifiable domain where Theorem 6 holds. Up to now, we have been able to prove Theorem 7 in strongly convex domains ([A1]), and in several weakly convex circular domains ([A4, AT]); therefore, being a local statement, it also holds in strongly pseudoconvex domains ([A3]) and, more generally, in domains whose boundary is locally biholomorphic to the boundary of a "good" 
domain. In all cases, we use the localized version (Example 3) of the canonical projection device (Example 2).

Actually, the complete statement of Theorem 7 in each case is more precise. For instance, there is always a direction $\nu_{x}$ (the "holomorphic normal direction"; usually, $\left.\nu_{x}=\varphi_{x}^{\prime}(1)\right)$ such that the limit of $\partial f / \partial \nu_{x}$ is explicitly computable. Or, if $D=\Delta^{n}$ and $v$ is tangent to a flat side of $\partial \Delta^{n}$ at $x$, then the limit is 0 , as well as when $D$ is strongly convex and $v$ is complex tangential to $\partial D$ at $x$.

Furthermore, there are similar theorems for holomorphic maps with values in a second domain $D^{\prime} \subset \subset \mathbb{C}^{m}$, at least when $D^{\prime}$ is strongly (pseudo)convex. For instance, if $f: \Delta \rightarrow D^{\prime}$ satisfies the analogue of $(4)$ at a point $\sigma_{0} \in \partial \Delta$, then $d(\zeta, \partial \Delta)^{1-s}\left(f^{\prime}, v\right)$ has non-tangential limit at $\sigma_{0}$, where $v \in \mathbb{C}^{m}, s$ is defined as in $(5)$, and $(\cdot, \cdot)$ is the canonical hermitian product on $\mathbb{C}^{m}$.

The main trick in the proof is to replace the non-holomorphic function $d(z, \partial D)$ by a holomorphic one of the form $1-\tilde{p}_{x}(z)$, where $\tilde{p}_{x} \in \operatorname{Hol}(D, \Delta)$ is such that

$$
1-\tilde{p}_{x}(z)=O(d(z, \partial D))
$$

in $K$-regions. If $D$ is convex, we use $\tilde{p}_{x}=\varphi_{x}^{-1} \circ p_{x}$. Since, in all the worked out cases, either $s=1$ or the limit (6) is zero, the replacement of $d(z, \partial D)$ by $1-\tilde{p}_{x}(z)$ does not change the content of Theorem 7 .

The proof then has two parts: first we show that $\left(1-\tilde{p}_{x}\right)^{s-1} \partial f / \partial v$ is $K$-bounded; and next we show that $\left(1-\tilde{p}_{x}\right)^{s-1} \partial f / \partial v$ has limit along a given special restricted $x$-curve, usually $t \mapsto \varphi_{x}(t)$.

We end this paper describing the first part of the proof of Theorem 7 when $D$ is a convex circular domain - a special case where the main ideas are not obscured by too many technical details.

A domain $D \subset \subset \mathbb{C}^{n}$ is circular if $z \in D$ implies $e^{i \theta} z \in D$ for all $\theta \in \mathbb{R}$. In particular, a convex circular bounded domain $D$ is the unit ball for a norm $\|\cdot\| \cdot \|$. It follows (see $[$ A2, JP]) that

$$
d(z, \partial D)=1-\|z\|
$$

and

$$
k_{D}(0, z)=\frac{1}{2} \log \frac{1+\|\mid\| z \|}{1-\||\| z|} .
$$

Fix $x \in \partial D$; we shall use the canonical projection device, choosing $\varphi_{x}(\zeta)=\zeta x$. Lempert's results $([\mathrm{L}]$; see also $[\mathrm{A} 2, \mathrm{JP}])$ provide us with a holomorphic retraction $p_{x}: D \rightarrow \varphi_{x}(\Delta)$; set $\tilde{p}_{x}=\varphi_{x}^{-1} \circ p_{x}$. Of course, the pole of all $K$-regions will be the origin.

First of all we must show that the function $\left(1-\tilde{p}_{x}(z)\right) / d(z, \partial D)$ is $K$-bounded at $x$. In fact, it is easy to check that if $z \in K_{0}(x, M)$ then $\tilde{p}_{x}(z)$ belongs to the Stolz region $S(1, M)$, and thus

$$
\left|1-\tilde{p}_{x}(z)\right| \leq M\left(1-\left|\tilde{p}_{x}(z)\right|\right)=M\left(1-\left\|p_{x}(z)\right\|\right),
$$

where the last equality follows from $p_{x}=\varphi_{x} \circ \tilde{p}_{x}$ and (7). Now, an easy argument (see [A1, Lemma 3.11]) using the contracting property of the Kobayashi distance shows that

$$
\forall z \in K_{0}(x, M) \quad 0 \leq k_{D}(0, z)-k_{D}\left(0, p_{x}(z)\right) \leq \log M
$$


therefore it follows that

$$
1-\left\|p_{x}(z)\right\| \mid \leq M^{2}(1-\||\|\mid\|)
$$

and thus

$$
\forall z \in K_{0}(x, M) \quad\left|1-\tilde{p}_{x}(z)\right| \leq M^{3} d(z, \partial D),
$$

as claimed. Notice that $k_{D}(0, z) \geq k_{\Delta}\left(0, \tilde{p}_{x}(z)\right)$ implies

$$
\left|1-\tilde{p}_{x}(z)\right| \geq d(z, \partial D)
$$

always. So we can (almost) forget $d(z, \partial D)$ and work with $1-\tilde{p}_{x}(z)$ only.

Now let $f \in \operatorname{Hol}(D, \Delta)$ be such that (4) holds. In particular, $f$ has $K$-limit $\tau \in \partial \Delta$ at $x$. Fix $v \in \mathbb{C}^{n}$. For every $z \in D$ let $\psi_{z} \in \operatorname{Hol}(\Delta, D)$ be a complex geodesic with $\psi_{z}(0)=z$ and $\psi_{z}^{\prime}(0)=v / \kappa_{D}(z ; v)$. Choose $r \in(0,1)$. Then

$$
\begin{aligned}
(1- & \left.\tilde{p}_{x}(z)\right)^{s-1} \frac{\partial f}{\partial v}(z)=\left(1-\tilde{p}_{x}(z)\right)^{s-1} \kappa_{D}(z ; v)\left(f \circ \psi_{z}\right)^{\prime}(0) \\
& =\left(1-\tilde{p}_{x}(z)\right)^{s-1} \frac{\kappa_{D}(z ; v)}{2 \pi i} \int_{|\zeta|=r} \frac{f\left(\psi_{z}(\zeta)\right)}{\zeta^{2}} d \zeta \\
& =\frac{1}{2 \pi} \int_{0}^{2 \pi} \frac{f\left(\psi_{z}\left(r e^{i \theta}\right)\right)-\tau}{\tilde{p}_{x}\left(\psi_{z}\left(r e^{i \theta}\right)\right)-1} \cdot \frac{\tilde{p}_{x}\left(\psi_{z}\left(r e^{i \theta}\right)\right)-1}{\tilde{p}_{x}(z)-1} \cdot\left(\frac{\tilde{p}_{x}(z)-1}{d(z, \partial D)}\right)^{s} \cdot \frac{d(z, \partial D)^{s} \kappa_{D}(z ; v)}{r e^{i \theta}} d \theta .
\end{aligned}
$$

We must show that the four factors in the integrand are $K$-bounded.

The fourth factor is bounded in $K$-regions by assumption, and we have already shown that the third factor is $K$-bounded too. To prove the $K$-boundedness of the remaining two factors we need three lemmas.

Lemma 8: Let $D \subset \subset \mathbb{C}^{n}$ be a convex circular domain, and take $x \in \partial D$ and $z \in D$. Then

$$
\limsup _{w \rightarrow x}\left[k_{D}(z, w)-k_{D}(0, w)\right]=\lim _{t \rightarrow 1^{-}}\left[k_{D}\left(z, \varphi_{x}(t)\right)-k_{\Delta}(0, t)\right],
$$

where $\varphi_{x}(t)=t x$.

Proof: First of all, it is easy to check that the function

$$
t \mapsto k_{D}\left(z, \varphi_{x}(t)\right)-k_{\Delta}(0, t)
$$

is not increasing for $t \in[0,1)$; let $h_{z}(x)$ denote its limit as $t \rightarrow 1^{-}$. By definition,

$$
h_{z}(x) \leq \limsup _{w \rightarrow x}\left[k_{D}(z, w)-k_{D}(0, w)\right]
$$

to prove the converse inequality we need to show that for every $\varepsilon>0$ there is $\delta>0$ so that if $\|w-x\|<\delta$ then

$$
k_{D}(z, w)-k_{D}(0, w)<h_{z}(x)+\varepsilon
$$


Choose $t_{0}<1$ so that

$$
k_{D}\left(z, \varphi_{x}(t)\right)-k_{\Delta}(0, t) \leq k_{D}\left(z, \varphi_{x}\left(t_{0}\right)\right)-k_{\Delta}\left(0, t_{0}\right) \leq h_{z}(x)+\varepsilon / 2
$$

and then fix $\delta_{0}>0$ such that if $y \in \partial D$ and $\|y-x\|<\delta_{0}$ then

$$
\left|k_{D}\left(z, t_{0} y\right)-k_{D}\left(z, t_{0} x\right)\right|<\varepsilon / 2 \text {. }
$$

Now choose $\delta>0$ so that $\|w-x\|<\delta$ implies both $\|w\|>t_{0}$ and

$$
\left\|\frac{w}{\|w w\|}-x\right\|<\delta_{0},
$$

where $\|\cdot\| \|$ is the norm associated to $D$. Then

$$
\begin{aligned}
k_{D}(z, w)-k_{D}(0, w) & =k_{D}\left(z,\|w\| \cdot \frac{w}{\|w\|}\right)-k_{\Delta}(0,\|w\| \|) \\
& \leq k_{D}\left(z, t_{0} \frac{w}{\|w\|}\right)-k_{\Delta}\left(0, t_{0}\right) \\
& \leq k_{D}\left(z, t_{0} x\right)-k_{\Delta}\left(0, t_{0}\right)+\varepsilon / 2 \\
& \leq h_{z}(x)+\varepsilon
\end{aligned}
$$

and we are done.

Lemma 9: Let $D \subset \subset \mathbb{C}^{n}$ be a convex circular domain; take $x \in \partial D$ and $f \in \operatorname{Hol}(D, \Delta)$. Then

$$
\liminf _{z \rightarrow x}\left[k_{D}(0, z)-k_{\Delta}(0, f(z))\right]=\lim _{t \rightarrow 1^{-}}\left[k_{\Delta}(0, t)-k_{\Delta}\left(0, f\left(\varphi_{x}(t)\right)\right)\right]
$$

where $\varphi_{x}(t)=t x$.

Proof: First of all, it is easy to check that the function

$$
t \mapsto k_{\Delta}(0, t)-k_{\Delta}(0, f(t x))
$$

is not decreasing in $[0,1)$; let $h \in \mathbb{R} \cup\{+\infty\}$ denote its limit as $t \rightarrow 1^{-}$. Clearly,

$$
\liminf _{z \rightarrow x}\left[k_{D}(0, z)-k_{\Delta}(0, f(z))\right] \leq h
$$

to prove the converse inequality we need to show that for every $\varepsilon>0$ there is a $\delta>0$ so that $\|z-x\|<\delta$ implies

$$
k_{D}(0, z)-k_{\Delta}(0, f(z)) \geq h-\varepsilon
$$

Choose $t_{0}>0$ so that

$$
\forall t \geq t_{0} \quad k_{\Delta}(0, t)-k_{\Delta}(0, f(t x)) \geq h-\varepsilon / 2,
$$


and take $\delta_{0}>0$ so that if $y \in \partial D$ is such that $\|y-x\|<\delta_{0}$ then

$$
\left|k_{\Delta}\left(0, f\left(t_{0} x\right)\right)-k_{\Delta}\left(0, f\left(t_{0} y\right)\right)\right|<\varepsilon / 2 .
$$

In particular,

$$
\begin{aligned}
k_{\Delta}(0, t)-k_{\Delta}(0, f(t y)) & \geq k_{\Delta}\left(0, t_{0}\right)-k_{\Delta}\left(0, f\left(t_{0} y\right)\right) \\
& \geq k_{\Delta}\left(0, t_{0}\right)-k_{\Delta}\left(0, f\left(t_{0} x\right)\right)-\varepsilon / 2 \geq h-\varepsilon
\end{aligned}
$$

for all $t \geq t_{0}$.

Then choose $\delta>0$ so that $\|z-x\|<\delta$ implies $\|z\|>t_{0}$ and

$$
\left\|\frac{z}{\|z\| \mid}-x\right\|<\delta_{0}
$$

Then

$$
k_{D}(0, z)-k_{\Delta}(0, f(z))=k_{\Delta}(0,\|z\| \|)-k_{\Delta}\left(0, f\left(\|z\| \cdot \frac{z}{\|z\|}\right)\right) \geq h-\varepsilon,
$$

and we are done.

Lemma 10: Let $D \subset \subset \mathbb{C}^{n}$ be a convex circular domain; take $x \in \partial D$. For $M_{1}>M>1$ set $r=\left(M_{1}-M\right) /\left(M_{1}+M\right)<1$. If $z \in K_{0}(x, M)$ and $\psi_{z} \in \operatorname{Hol}(\Delta, D)$ is a complex geodesic such that $\psi_{z}(0)=z$ then $\psi_{z}\left(\Delta_{r}\right) \subseteq K_{0}\left(x, M_{1}\right)$, where $\Delta_{r}=\{\zeta \in \mathbb{C}|| \zeta \mid<r\}$.

Proof: Let $\delta=\frac{1}{2} \log \left(M_{1} / M\right)>0$; then $\zeta \in \Delta_{r}$ iff $k_{\Delta}(0, \zeta)<\delta$. Then (by Lemma 8)

$$
\begin{aligned}
\limsup _{w \rightarrow x}\left[k_{D}\left(\psi_{z}(\zeta), w\right)\right. & \left.-k_{D}(0, w)\right]+k_{D}\left(0, \psi_{z}(\zeta)\right) \\
& =\lim _{t \rightarrow 1^{-}}\left[k_{D}\left(\psi_{z}(\zeta), t x\right)-k_{\Delta}(0, t)\right]+k_{D}\left(0, \psi_{z}(\zeta)\right) \\
& \leq 2 k_{D}\left(\psi_{z}(\zeta), z\right)+\lim _{t \rightarrow 1^{-}}\left[k_{D}(z, t x)-k_{\Delta}(0, t)\right]+k_{D}(0, z) \\
& <2 k_{\Delta}(0, \zeta)+\log M=\log M_{1} .
\end{aligned}
$$

This is what we need to prove the $K$-boundedness of the two remaining factors. Indeed, for the first one choose $z \in K_{0}(x, M)$ and set

$$
\frac{1}{2} \log R=\log M-k_{D}(0, z)
$$

in particular, being $k_{D}(0, z) \geq k_{\Delta}\left(0, \tilde{p}_{x}(z)\right)$, we have

$$
R \leq M^{2} \frac{1-\left|\tilde{p}_{x}(z)\right|}{1+\left|\tilde{p}_{x}(z)\right|} \leq M^{2}\left|1-\tilde{p}_{x}(z)\right|
$$


Let

$$
\frac{1}{2} \log \alpha=\liminf _{z \rightarrow x}\left[k_{D}(0, z)-k_{\Delta}(0, f(z))\right]=\lim _{t \rightarrow 1^{-}}\left[k_{\Delta}(0, t)-k_{\Delta}(0, f(t x))\right],
$$

where the latter equality follows from Lemma 9 , and let $\tau \in \partial \Delta$ be the $K$-limit of $f$ at $x$. Then

$$
\begin{aligned}
\log \frac{|\tau-f(z)|}{1+|f(z)|} & =\lim _{t \rightarrow 1^{-}}\left[k_{\Delta}(f(z), t \tau)-k_{\Delta}(0, t \tau)\right]-k_{\Delta}(0, f(z)) \\
& \leq 2 \lim _{t \rightarrow 1^{-}}\left[k_{\Delta}(f(z), f(t x))-k_{\Delta}(0, f(t x))\right] \\
& \leq 2 \lim _{t \rightarrow 1^{-}}\left[k_{D}(z, t x)-k_{\Delta}(0, f(t x))\right] \\
& =2 \lim _{t \rightarrow 1^{-}}\left[k_{D}(z, t x)-k_{\Delta}(0, t)\right]+2 \lim _{t \rightarrow 1^{-}}\left[k_{\Delta}(0, t)-k_{\Delta}(0, f(t x))\right] \\
& \leq \log (\alpha R),
\end{aligned}
$$

(where the second inequality holds because $f(t x) \rightarrow \tau$ as $t \rightarrow 1^{-}$) and we get

$$
\left|\frac{\tau-f(z)}{1-\tilde{p}_{x}(z)}\right| \leq 2 \alpha M^{2}
$$

In particular, by Lemma 10 we get

$$
\left|\frac{\tau-f\left(\psi_{z}\left(r e^{i \theta}\right)\right)}{1-\tilde{p}_{x}\left(\psi_{z}\left(r e^{i \theta}\right)\right)}\right| \leq 2 \alpha M_{1}^{2},
$$

where $M_{1}>M$ is such that $\left(M_{1}-M\right) /\left(M_{1}+M\right)=r$, and the first factor is $K$-bounded.

For the second factor, we have already remarked that $\psi_{z}\left(r e^{i \theta}\right) \in K_{0}\left(x, M_{1}\right)$ implies $\tilde{p}_{x}\left(\psi_{z}\left(r e^{i \theta}\right)\right) \in S\left(1, M_{1}\right)$, and so

$$
\left|\frac{1-\tilde{p}_{x}\left(\psi_{z}\left(r e^{i \theta}\right)\right)}{1-\tilde{p}_{x}(z)}\right| \leq M_{1} \frac{1-\left\|p_{x}\left(\psi_{z}\left(r e^{i \theta}\right)\right)\right\| \|}{1-\left\|p_{x}(z)\right\|} .
$$

Now,

$$
\begin{aligned}
\frac{1}{2} \log \frac{1-\left\|p_{x}\left(\psi_{z}\left(r e^{i \theta}\right)\right)\right\| \|}{2\left(1-\left\|p_{x}(z)\right\|\right)} & \leq\left|k_{D}\left(0, p_{x}(z)\right)-k_{D}\left(0, p_{x}\left(\psi_{z}\left(r e^{i \theta}\right)\right)\right)\right| \\
& \leq k_{D}\left(p_{x}(z), p_{x}\left(\psi_{z}\left(r e^{i \theta}\right)\right)\right) \leq k_{D}\left(z, \psi_{z}\left(r e^{i \theta}\right)\right)=k_{\Delta}(0, r),
\end{aligned}
$$

and the second factor is $K$-bounded too.

To finish the proof of Theorem 7 (assuming of course that Theorem 6 holds) one should show that $\left(1-\tilde{p}_{x}(z)\right)^{1-s} \partial f / \partial v$ admits limit along a special restricted $x$-curve. If $v=x=\varphi_{x}^{\prime}(1)$, using the curve $\gamma(t)=\varphi_{x}(t)$ we are reduced to a one-dimensional situation, and the assertion follows from Theorem 2. In general, if $D$ is Reinhardt (that is, $z=\left(z_{1}, \ldots, z_{n}\right) \in D$ implies $\left(e^{i \theta_{1}} z_{1}, \ldots, e^{i \theta_{n}} z_{n}\right) \in D$ for all $\left.\theta_{1}, \ldots, \theta_{n} \in \mathbb{R}\right)$ we can use the argument of [A4, Proposition 4.9] to show that $\partial f / \partial v$ has limit along the given curve $\gamma$, and thus Theorem 7 follows if $s \geq 1$. If $s<1$ (or $D$ is not Reinhardt), a case-by-case argument is again needed; see, for instance, [A1, Proposition 3.19]. 


\section{References}

[A1] M. Abate: The Lindelöf principle and the angular derivative in strongly convex domains. J. Analyse Math. 54 189-228 (1990)

[A2] M. Abate: Iteration theory of holomorphic maps on taut manifolds. Mediterranean Press, Rende, 1989

[A3] M. Abate: Angular derivatives in strongly pseudoconvex domains. Proc. Symp. Pure Math. 52, Part 2, 23-40 (1991)

[A4] M. Abate: The Julia-Wolff-Carathéodory theorem in polydisks. To appear in J. Analyse Math. 74 (1998)

[AT] M. Abate, R. Tauraso: Angular derivatives in complex ellipsoids. In preparation, 1998

[C] C. Carathéodory: Über die Winkelderivierten von beschränkten analytischen Funktionen. Sitzungsber. Preuss. Akad. Wiss., Berlin 39-54 (1929)

[CK] J.A. Cima, S.G. Krantz: The Lindelöf principle and normal functions of several complex variables. Duke Math. J. 50 303-328 (1983)

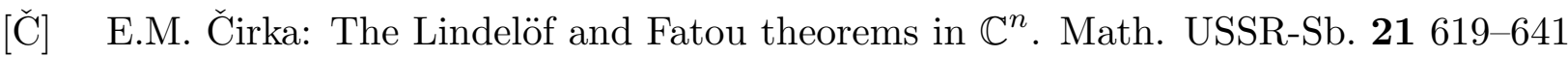
(1973)

[F] P. Fatou: Séries trigonométriques et séries de Taylor. Acta Math. 30 335-400 (1906)

[H] M. Hervé: Quelques propriétés des applications analytiques d'une boule à $m$ dimensions dans elle-même. J. Math. Pures Appl. 42 117-147 (1963)

[J] F. Jafari: Angular derivatives in polydisks. Indian J. Math. 35 197-212 (1993)

[JP] M. Jarnicki, P. Pflug: Invariant distances and metrics in complex analysis. Walter de Gruyter, Berlin, 1993

[Ju] G. Julia: Extension nouvelle d'un lemme de Schwarz. Acta Math. 42 349-355 (1920)

[K] A. Korányi: Harmonic functions on hermitian hyperbolic spaces. Trans. Amer. Math. Soc. 135 507-516 (1969)

[KS] A. Korányi, E.M. Stein: Fatou's theorem for generalized half-planes. Ann. Scuola Norm. Sup. Pisa 22 107-112 (1968)

[Kr] S.G. Krantz: Invariant metrics and the boundary behavior of holomorphic functions on domains in $\mathbb{C}^{n}$. J. Geom. Anal. 1 71-97 (1991)

[L] L. Lempert: La métrique de Kobayashi et la représentation des domaines sur la boule. Bull. Soc. Math. France 109 427-474 (1981)

[Li] E. Lindelöf: Sur un principe générale de l'analyse et ses applications à la theorie de la représentation conforme. Acta Soc. Sci. Fennicae 46 1-35 (1915)

[R] W. Rudin: Function theory in the unit ball of $\mathbb{C}^{n}$. Springer, Berlin 1980

[S] E.M. Stein: The boundary behavior of holomorphic functions of several complex variables. Princeton University Press, Princeton, 1972

[W] J. Wolff: Sur une généralisation d'un théorème de Schwarz. C.R. Acad. Sci. Paris 183 500-502 (1926) 